\title{
Switch-on Phenomena and Field Emission from Multi-Walled Carbon Nanotubes Embedded in Glass
}

*Correspondence to:

Mousa MS,

Tel: +962-79-565-9761

Fax: +962-3-2375540

E-mail: marwansmousa@yahoo.com

Received July 20, 2016

Revised October 8, 2016

Accepted November 10, 2016

\author{
Emad S Bani Ali, Marwan S Mousa* \\ Department of Physics, Mu'tah University, Al-Karak 61710, Jordan
}

\begin{abstract}
This paper describes a new design of carbon nanotube tip. Nanocly ${ }^{\mathrm{TM}}$ NC 7000 Thin Multiwall Carbon Nanotubes of carbon purity (90\%) and average diameter tube $9.5 \mathrm{~nm}$ with a high aspect-ratio $(>150)$ were used. These tips were manufactured by employing a drawing technique using a glass puller. The glass microemitters with internal carbon nanotubes show a switch-on effect to a high current level (1 to $20 \mu \mathrm{A})$. A field electron microscope with a tip (cathode)-screen (anode) separation at $\sim 10 \mathrm{~mm}$ was used to characterize the electron emitters. The system was evacuated down to a base pressure of $\sim 10^{-9}$ mbar when baked at up to $\sim 200^{\circ} \mathrm{C}$ overnight. This allowed measurements of typical Field Electron Emission characteristics; namely the current-voltage $(I-V)$ characteristics and the emission images on a conductive phosphorus screen (the anode). FowlerNordheim plots of the current-voltage characteristics show current switch-on for each of these emitters.
\end{abstract}

Key Words: Carbon nanotubes, Cold field electron emission, Field electron microscope, Fowler-Nordheim plots, Glass microemitters

\section{INTRODUCTION}

Cold field electron emission (CFE) is an electron emission regime where electrons are field emitted from a surface, by tunneling from states near the emitter Fermi level (Forbes, 2012; Forbes et al., 2015). The emission process is controlled by the height and width of the surface potential-energy (PE) barrier, and the barrier width is determined by the magnitude $F$ of the local barrier electrostatic field. For metal emitters, the emission current $I$ from a single tip emitter is given by a Fowler-Nordheim-type (FN-type) equation (Forbes, 2012; Forbes et al., 2015):

$$
I=A_{\mathrm{f}} a \phi^{-1} F^{2} \exp \left[-v_{\mathrm{F}} b \phi^{3 / 2} / F\right]
$$

where $a$ and $b$ are the first and second FN constants, $\phi$ is the local work-function, $v_{\mathrm{F}}$ is the barrier-form correction factor for the particular potential-barrier shape assumed, and $A_{\mathrm{f}}$ is the formal emission area, as defined in (Forbes et al., 2015). It is often assumed that this equation is also an adequate approximation for emission from non-metallic emitters. Carbon nanotubes (CNTs) (Iijima, 1991) are comprised of graphene sheets rolled into seamless hollow cylinders with diameters ranging from $1 \mathrm{~nm}$ to about $50 \mathrm{~nm}$ (Saito et al., 1998). CNTs have the following properties that make them appropriate as CFE sources: (1) high aspect ratio, (2) small apex radius of curvature, (3) high chemical stability, and (4) high mechanical strength (Bonard et al., 1999; Choi et al., 1999; Gröning et al., 2000; Saito et al., 1998; Saito \& Uemura, 2000). This study uses Nanocyl ${ }^{\mathrm{TM}}$ NC7000 (Nanocyl S. A., Belgium) thin multi-walled carbon nanotubes (MWCNTs) with $9.5 \mathrm{~nm}$ diameter and high aspect ratio $(>150)$, fabricated by chemical vapor deposition (Bonard et al., 1998, 2002; de Jonge \& Bonard, 2004).

Individual MWCNTs (Kim, 2013) were melt-welded into a fine glass capillary tube, using a "glass puller" of the form

(a) This is an open-access article distributed under the terms of the Creative Commons Attribution Non-Commercial License (http://creativecommons.org/licenses/by-nc/4.0) which permits unrestricted noncommercial use, distribution, and reproduction in any medium, provided the original work is properly cited.

Copyrights @ 2016 by Korean Society of Microscopy 


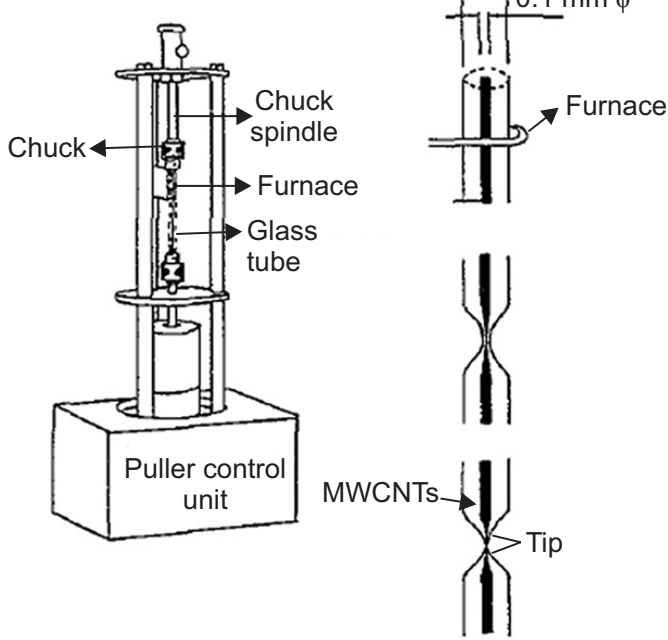

Fig. 1. Schematic diagram of the glass puller. MWCNTs, multi-walled carbon nanotubes.

shown in Fig. 1. This creates a prepared glass micro-point emitter (Mousa \& Hibbert, 1993a) (Fig. 1), which will field emit when a sufficiently high electric field is applied. In this work a new kind of emitter is introduced which is based on internally a MWCNTs enclosed within a glass microemitter. The composite emitter is fabricated using a pulling technique. The analyses were essentially carried out using a field emission microscope. This system could be evacuated to a base pressure of about $10^{-9} \mathrm{mbar}$ after baking at $\sim 200^{\circ} \mathrm{C}$ overnight. The cathode was mounted $\sim 10 \mathrm{~mm}$ away from the phosphorus screen and a current limiting resistor of $100 \mathrm{M} \Omega$ was used.

\section{MATERIALS AND METHODS}

As indicated, composite glass-on-MWCNTs emitters were prepared by the technique of pulling heated glass capillary tubes into fine points (Mousa \& Hibbert, 1993a), using the device shown in Fig. 1. In this technique, two bearings are located accurately on plates supported by three stainless steel rods fixed rigidly to the frame of the control unit. This frame is strong enough to serve as a stable base for the instrument. A glass tube (outside diameter $=1 \mathrm{~mm}$, internal diameter $=0.1$ $\mathrm{mm}$ ) fits inside these bearings, between the upper and lower chucks, with a furnace loop located around it. The MWCNTs were entered into the opposite end of each glass tube so they would protrude at the tip with a wire plunger (Mousa \& Hibbert, 1993b). The lower chuck spindle can slide vertically, in order to pull down the glass tube under gravity. In operation, the temperature of the furnace is raised to the glass softening point (1400 K for borosilicate glass) (Mousa, 1991). Prepared emitters were investigated in a locally built pointto-plane field electron miroscope (FEM), with an axial tip-
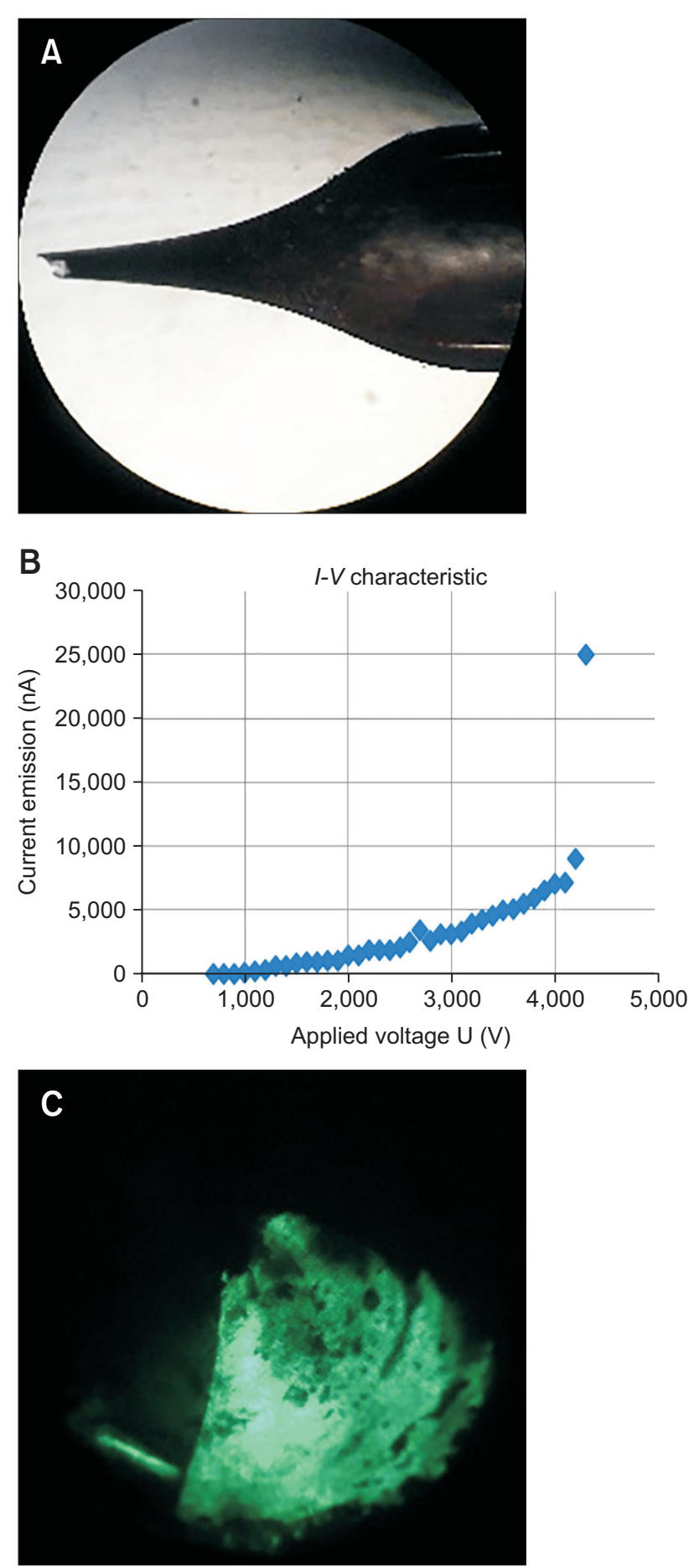

Fig. 2. (A) Optical micrograph of (CNT-A4) tip at 50X times magnification, allowing studies of the emitter (tip) profile. (B) The $I-V$ plot of this tip shows a switch-on phenomenon, at the voltage and current values $V_{\mathrm{sw}}=$ $4,300 \mathrm{~V}, I_{\mathrm{sW}}=25 \mu \mathrm{A}$. (C) Field electron miroscope image after switch-on.

to-screen distance of $10 \mathrm{~mm}$ (Al-Qudah et al., 2015; Mousa et al., 2015). The FEM was evacuated to ultra high vacuum conditions by the combination of a rotary pump (which produces a backing pressure of about $10^{-3} \mathrm{mbar}$ ) and an oil 
diffusion pump system, connected to a liquid nitrogen $\left(\mathrm{LN}_{2}\right)$ trap. Without baking, the FEM reaches a base-pressure of about $10^{-7}$ mbar. The pressure reaches about $10^{-9} \mathrm{mbar}$ after baking the system for 12 hours at $180^{\circ} \mathrm{C}$, and adding liquid nitrogen to the trap (Latham \& Salim, 1987; Madanat et al., 2015; Moran Meza et al, 2015; Mousa et al., 2015).

In operation, the FEM applied voltage $V$ is increased slowly until a "switch-on voltage" $V_{\mathrm{SW}}$ is reached, at which point the emission current suddenly "switches on". At $V_{\mathrm{SW}}$ the current increases rapidly from about a nanoampere to a much greater saturated value $I_{\mathrm{SAT}}$, as found previously (Latham \& Mousa, 1986). The current-voltage $(I-V)$ data yields a FN plot that has linear segments.

\section{RESULTS}

To obtain quantitative information about the microscopic properties of the emitting regime, the total electron emission current $I$, has been plotted as a function of the applied voltage $V$ between the emitter and the anode. Emission characterization will be presented as $I-V$ plots and as the related FN plots. Throughout the experiments, electron emission images have been recorded by means of a digital camera, in order to study the spatial distribution and stability of the emission current.

Fig. 2A shows an optical image of the first tip to be discussed. Fig. $2 \mathrm{~B}$ and $\mathrm{C}$ show the $I-V$ characteristic and a related FEM image. As we increased the applied voltage slowly, a point was reached, at voltage $V_{\mathrm{SW}}=4,300 \mathrm{~V}$, where the emission current suddenly 'switched-on' from an effective zero-value to a stable saturated value $I_{\mathrm{SAT}}$. Fig. $2 \mathrm{~B}$ shows the switch-on at $V_{\mathrm{SW}}=4,300$ $\mathrm{V}, I_{\mathrm{SW}}=25 \mu \mathrm{A}$. As voltage is then reduced, the "saturated" region extends down to the values $V_{\mathrm{SAT}}=1,900 \mathrm{~V}, I_{\mathrm{SAT}}=1 \mu \mathrm{A}$. Further voltage reduction causes the emission current to fall to nearly zero, reaching the threshold values $V_{\mathrm{TH}}=700 \mathrm{~V}$,

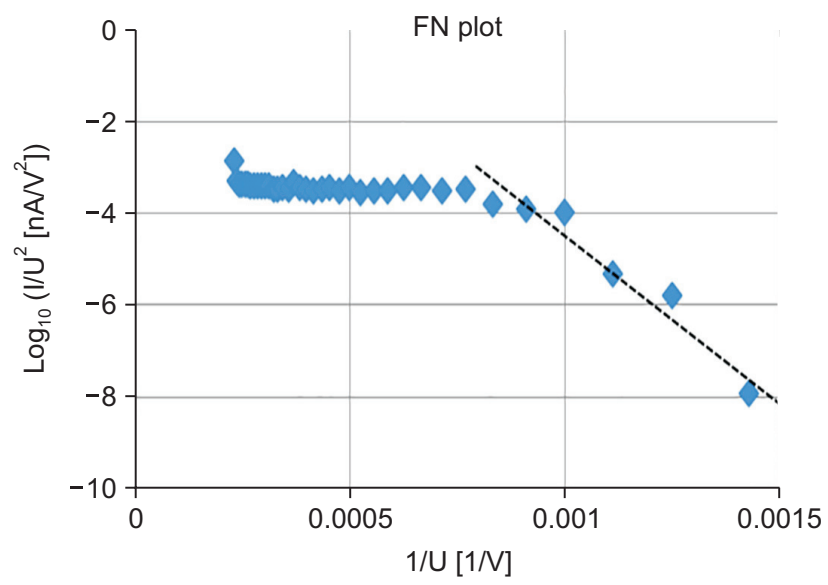

Fig. 3. Fowler-Nordheim (FN) plot of tip CNT-A4 showing linear trend at low field region.
$I_{\mathrm{TH}}=6.0 \times 10^{-12}$ A. As shown in Fig. 3, once the emitter leaves saturation, the (decreasing) current-voltage relationship generates an approximately linear FN plot, with slope about $-5,970$ decade $\mathrm{V}$.
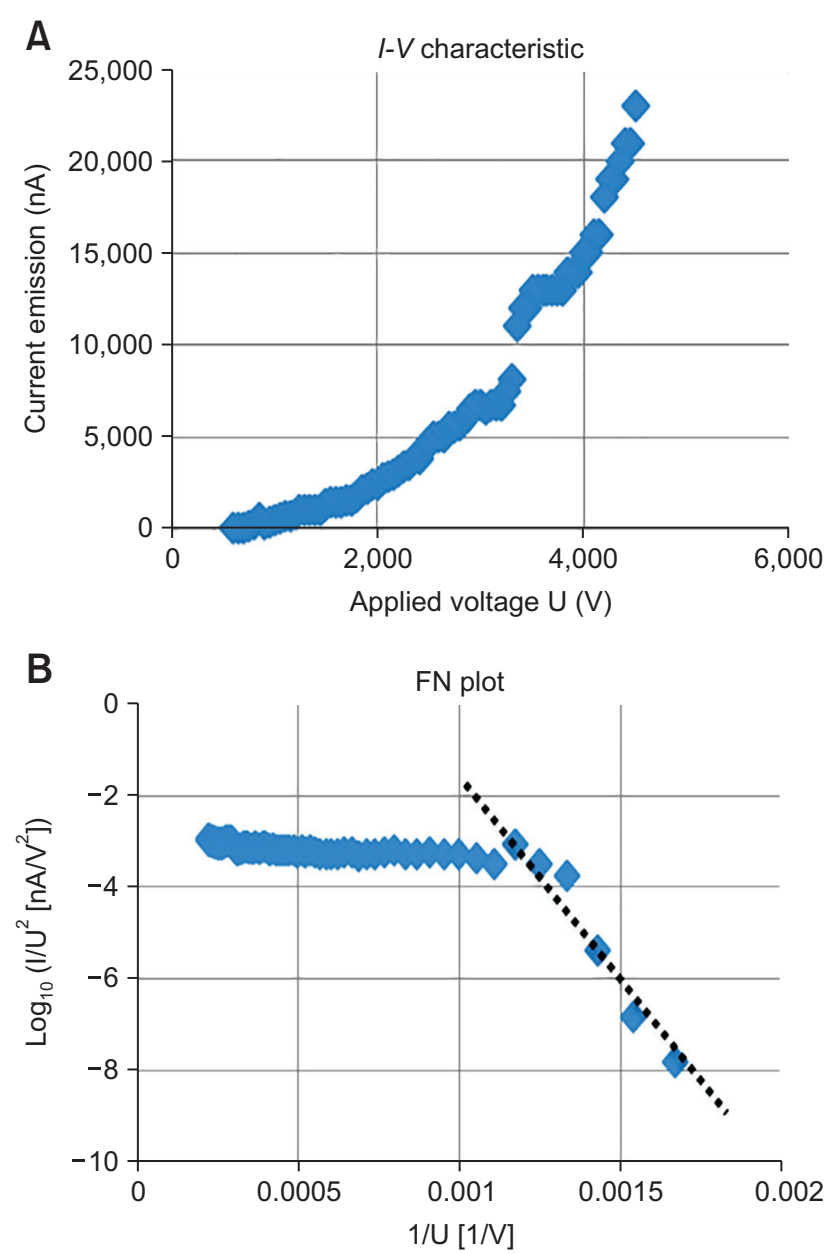

C

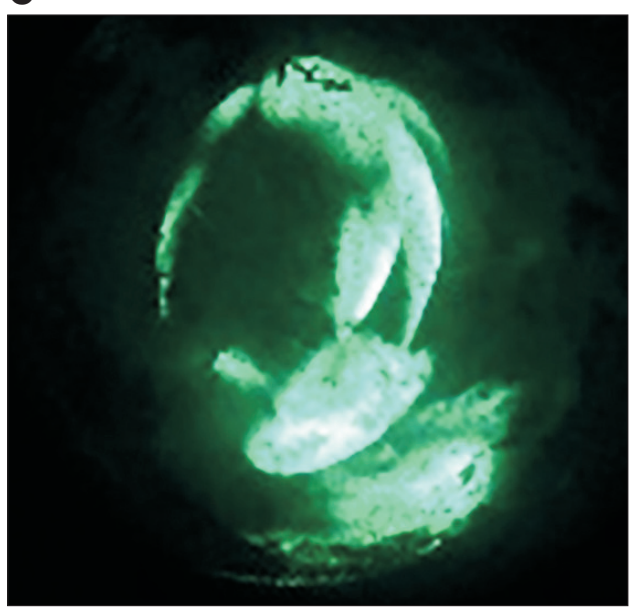

Fig. 4. (A) The $I-V$ plot of this tip shows switch-on phenomena $\left(V_{\mathrm{SW}}=4,500\right.$ $\mathrm{V}, I_{\mathrm{sW}}=23 \mu \mathrm{A}$ ). (B) Fowler-Nordheim (FN) plot. (C) Field electron miroscope images after switch-on. 
The experiment was then repeated after allowing "relaxation". (Relaxation is the name we give to the procedure whereby, after an initial experimental run, we turn off the voltage, and leave the sample under vacuum for 12 hours before repeating an experimental run [Mousa et al., 2015]). During this time the pressure increased to $4.9 \times 10^{-8}$ mbar. The current-voltage characteristics show initiation by low current emission at $V=4,400 \mathrm{~V}, I=1.3 \mathrm{nA}$. This is followed by switchon at $V_{\mathrm{SW}}=4,500 \mathrm{~V}, I_{\mathrm{SW}}=23 \mu \mathrm{A}$ to a saturated current $I_{\mathrm{SAT}}$ that may be seen in the expanded voltage range in Fig. 4 . The emission characteristics of this tip show a saturation effect for high emission current. The dashed line represents the data for the low field region, which yields a linear FN plot with slope $=-10,470$ decade $\mathrm{V}$ as shows in Fig. 4C.

As presented in Fig. 4C, the emission image showed large bright spots, spread over most of the screen.We then repeated the experiment, for the same tip (CNT-A4) and under similar conditions (but with a baking period of 14 hours).
The following figures (Fig. 5-9) show cycles of increasing and decreasing the applied voltage.

The emission images shown in Fig. 6 (when increasing the applied voltage) are more concentrated. However, the image became large and diffuse when decreasing the voltage as shown in Fig. 7C.

In Fig. 9, the emission images show a stable bright spot, but when the applied voltage was decreased and reached the point $V_{\mathrm{SW} 2}=2,800 \mathrm{~V}, I_{\mathrm{SW} 2}=5.8 \mu \mathrm{A}$ the emission current increased suddenly. In Fig. $8 \mathrm{~B}$, the $I-V$ plot shows evident suddenly increasing of emission current. Decreasing the applied voltage from $3,250 \mathrm{~V}$ to $1,000 \mathrm{~V}$ gave currents in the range $5.8 \mu \mathrm{A}$ down to $9.9 \mathrm{pA}$.

The shift of the emission current to lower values may occur because of surface impurities; alternatively, this shift could result from the presence of multiple emitting sites on the tip that switch on at different voltages (Mousa \& Hibbert, 1993b). Studies were also carried out on a second tip (CNT-A5). The
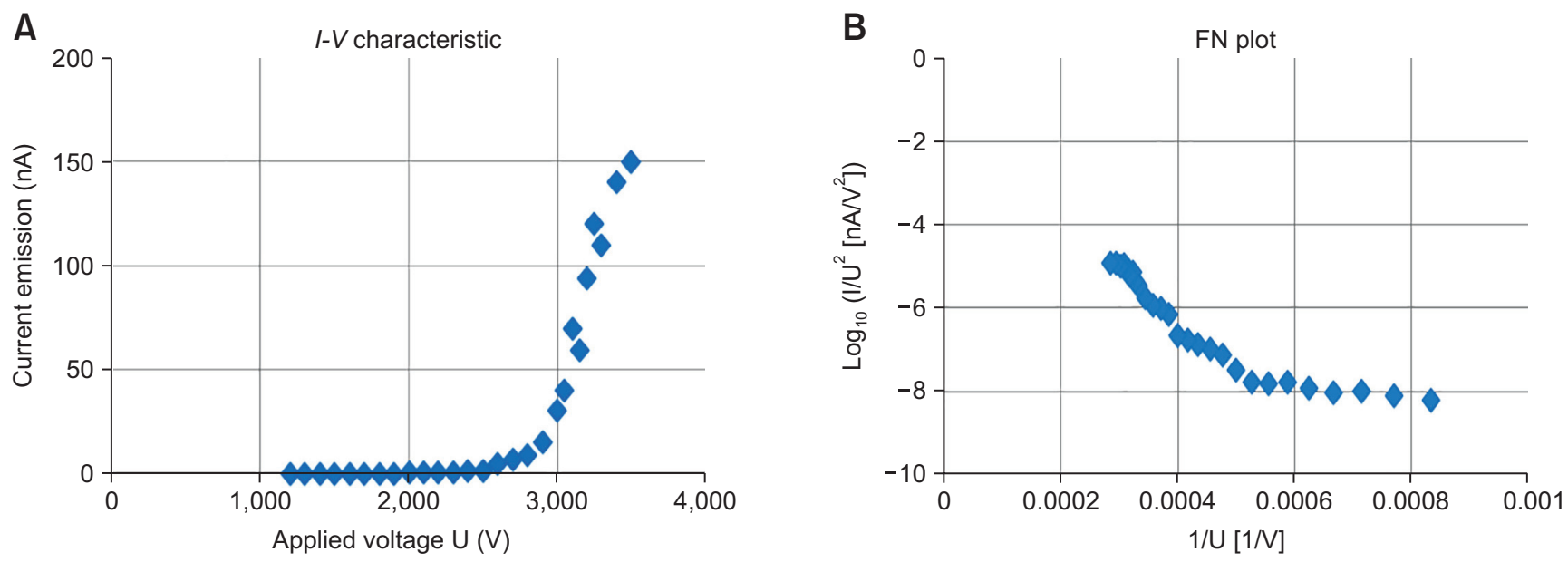

Fig. 5. Increasing voltage (A) $I-V$ plot $(1,200 \sim 3,500)$ V. (B) Fowler-Nordheim (FN) plot with slope about $-7,060$ decade V.
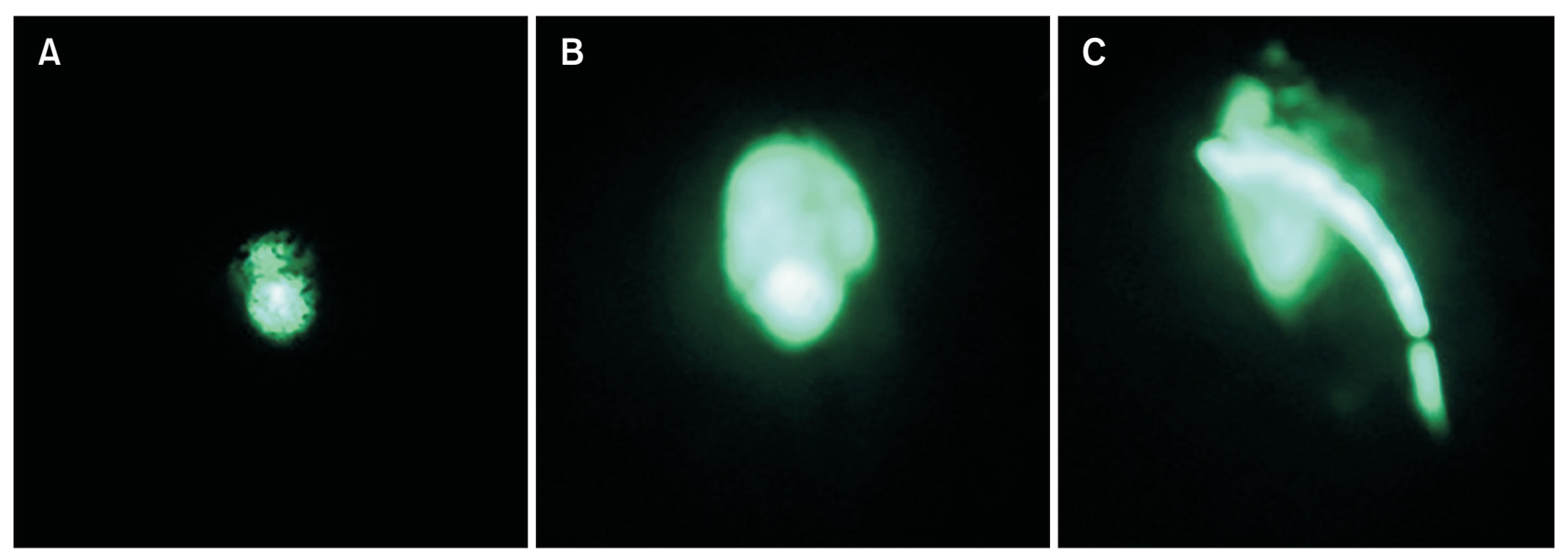

Fig. 6. Increasing voltage, emission images. (A) $V=3,500 \mathrm{~V}, I=0.15 \mu \mathrm{A}$. (B) $V=3,500 \mathrm{~V}, I=2.9 \mu \mathrm{A}$ (before switch-on). (C) $V_{\mathrm{sW}}=3,500 \mathrm{~V}, I_{\mathrm{SW}}=9.3 \mu \mathrm{A}$. 

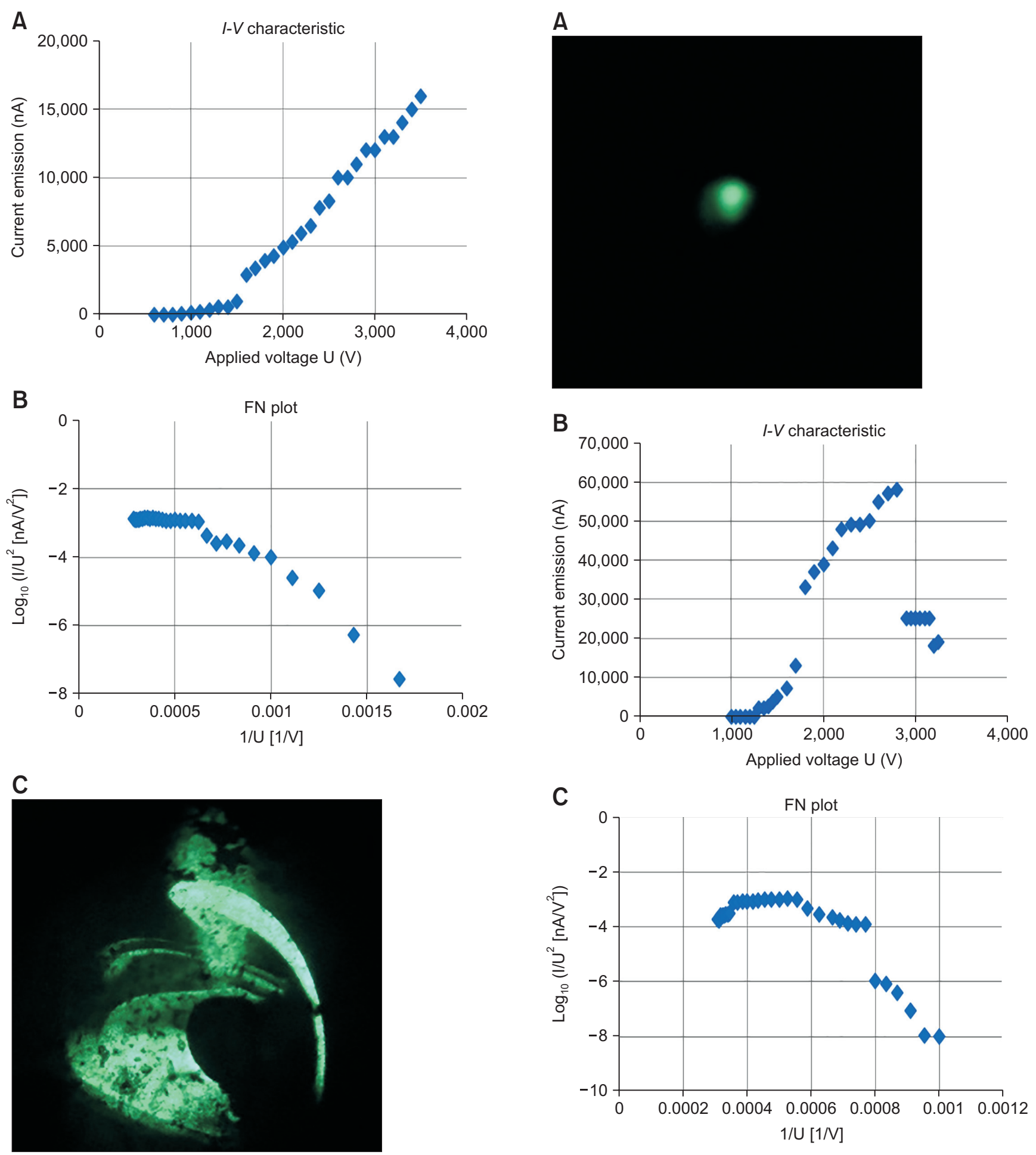

Fig. 7. Decreasing voltage. (A) $I-V$ plot $(3,500 \sim 600)$ V. (B) FowlerNordheim (FN) plot showing saturation for high emission current, linear FN plot in the low field region, with slope equal to about $-4,010$ decade $V$.

Fig. 8. Re-applied voltage again. (A) Emission image before switch-on $(V=3,050 \mathrm{~V}, I=0.6 \mu \mathrm{A})$, decreasing voltage. (B) $I-V$ plot $(3,250 \sim 1,000) \mathrm{V}$. (C) Fowler-Nordheim plot with slope about $-11,840$ decade V. 

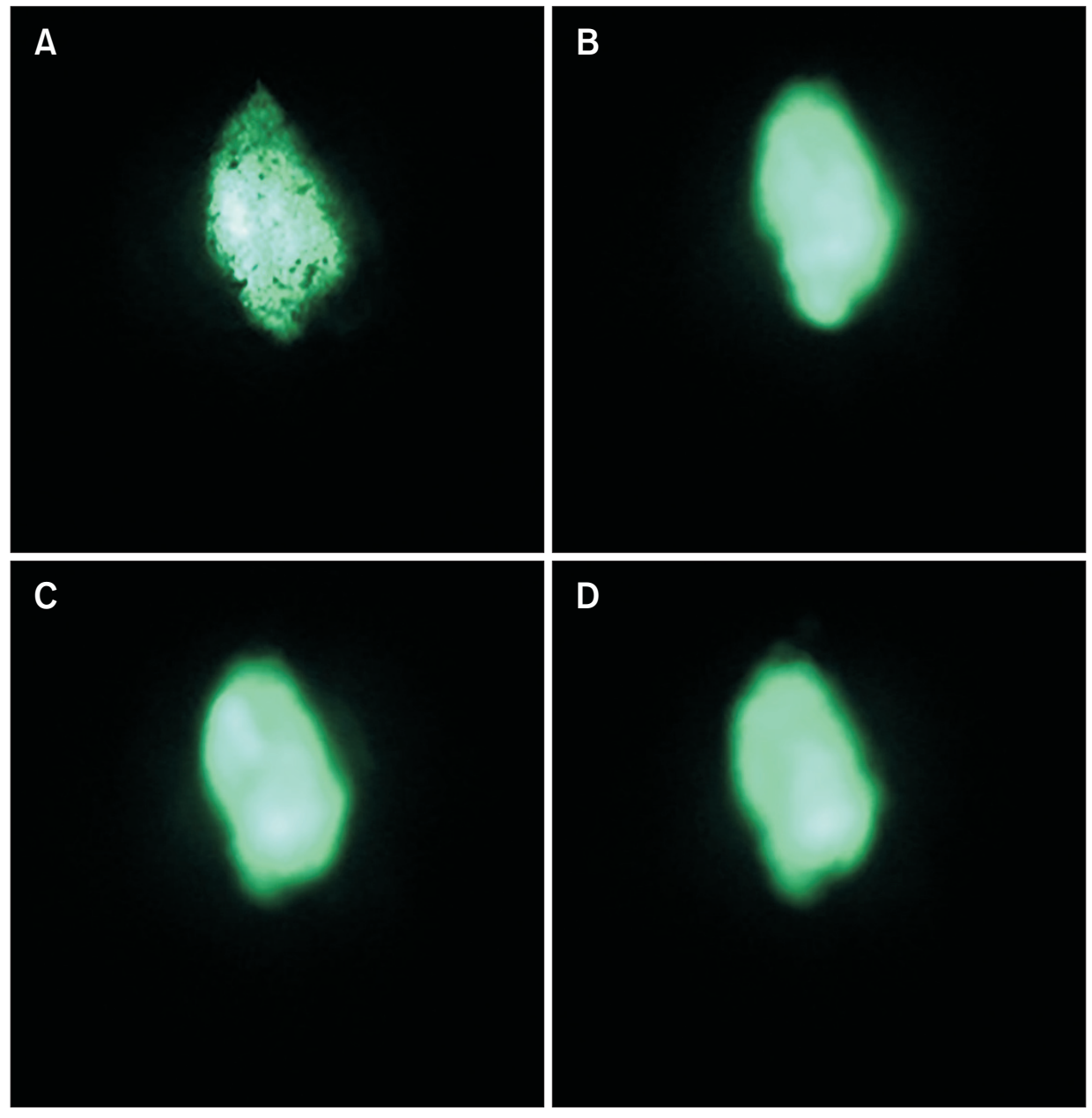

Fig. 9. Emission images recorded from third cycle, decreasing voltage. (A) $V_{\mathrm{sw1}}=$ $3,250 \mathrm{~V}, I_{\mathrm{SWl}}=1.9 \mu \mathrm{A}$ ). (B-D) Emission image at same emission current but different voltages $(3,150 \mathrm{~V}, 3,000 \mathrm{~V}, 2,900 \mathrm{~V})$.

optical micrograph in Fig. 10A shows the shape of this tip, which is a very smooth needle-like emitter. This tip has low switch-on voltage, as shown in its $I$ - $V$ characteristics and $\mathrm{FN}$ plot. With this tip, the voltage was cycled two times.

As the applied voltage is slowly increased the emission current "switches on" from $I=6.0 \mathrm{nA}$ to a stable saturated value of $3.2 \mu \mathrm{A}$ to $1.0 \mu \mathrm{A}$ by slowly reducing the voltage, te current decreases smoothly until it vanishes at a certain threshold voltage. Fig. 10B shows that for this emitter the switch-on occurs at $V_{\mathrm{SW}}=2,300 \mathrm{~V}, I_{\mathrm{SW}}=2.3 \mu \mathrm{A}$. The saturated region extends down to $V_{\mathrm{SAT}}=1,750 \mathrm{~V}, I_{\mathrm{SAT}}=1 \mu \mathrm{A}$. Beyond this, the emission current falls to nearly zero, as the applied voltage is decrease to a threshold value $V_{\mathrm{TH}}=1,430 \mathrm{~V}$, with $I_{\mathrm{TH}}=5.7 \mathrm{pA}$. In Fig. 10C shows FN plot showing saturation for high emission current, linear FN plot at the low field region with slope about $-8,720$ decade $\mathrm{V}$.

The emission images shown in Fig. 11 (when decreasing the applied voltage after switch-on) are more concentrated. The emission images in this case are small, concentrated bright and, stable. As shown in the related figures, a non-orthodox type of current-voltage behavior was observed with this emitter (R. Forbes; personal communication).

\section{DISCUSSION AND CONCLUSIONS}

It is helpful to attempt to understand the conditions under which continuous stable current may flow from the composite emitters. The electron emission dynamics is mostly controlled by the metal/glass interface (Mousa \& Hibbert, 1993b). Our measurements show that MWNTs have high current emission. However, our observations suggest that the presence of localized energy states at the tip, instead of the metallic density of states encountered on usual emitters, influences greatly the emission characteristics. The deviations from FN behavior and saturation effects, as well as the presence of discrete current levels, observed on single MWNTs are characteristic of materials with nonmetallic emitting states, as for example nanotips (Thien et al., 1996). Because of the nonmetallic nature of the tip, field penetration and fluctuations of the applied potential shift the position of the emitting levels. This could lead to the observed saturation at high applied voltages and strong instabilities of 

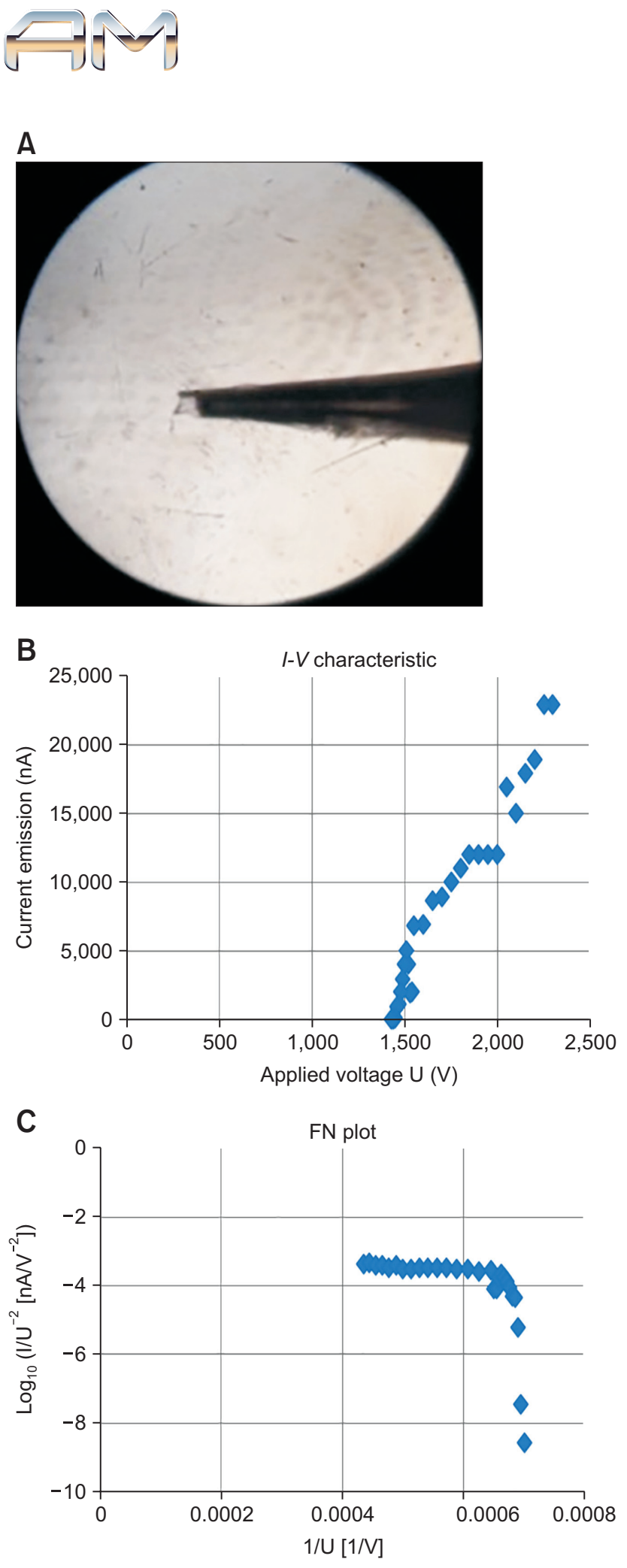

Fig. 10. (A) Optical micrograph of CNT-A5 tip at 50X times magnification, decreasing voltage. (B) $I-V$ plot with voltage range $(2,300 \sim 1,430) \mathrm{V}$ and current range (2.3 $\mu \mathrm{A}$ to $5.7 \mathrm{pA})$, (C) Fowler-Nordheim (FN) plot showing saturation for high emission current, linear FN at the low field region with slope about $-8,720$ decade $\mathrm{V}$.
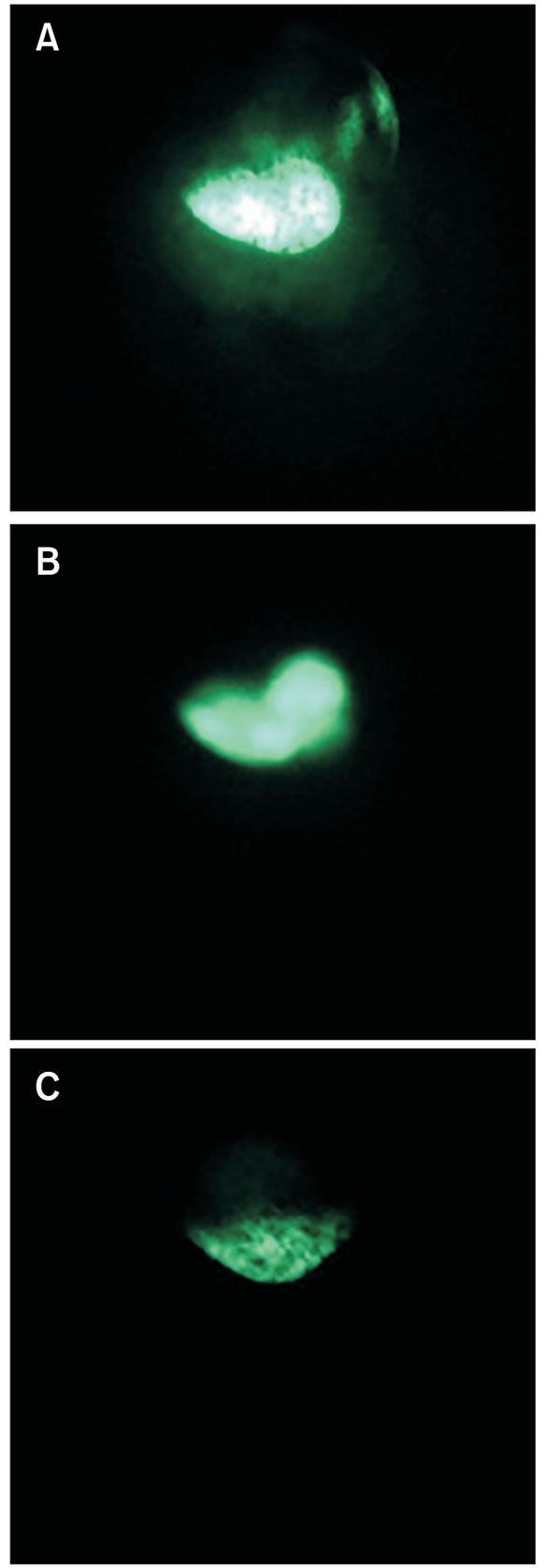

Fig. 11. Decreasing voltage, emission images. (A) $V_{\mathrm{sW}}=3,200 \mathrm{~V}, I_{\mathrm{sW}}=3.2 \mu \mathrm{A}$. (B) (after switch-on) $V=1,950 \mathrm{~V}, I=1.2 \mu \mathrm{A}$. (C) $V=1,510 \mathrm{~V}, I=0.5 \mu \mathrm{A}$.

the emission in some voltages ranges. The difference in energy between emitted and replacement electrons provokes a tip heating or cooling (Nottingham effect), related to the position of the Fermi level (Swanson \& Bell, 1973; Thien et al., 1996). On tubes with emitting states just above the Fermi level this reduces the increase of the temperature with the current. As for the current steps, they most likely reflect changes in the surface structure (Swanson \& Bell, 1973) and/or in the adsorbate coverage (adsorption, desorption or diffusion) 
(Lee et al., 1995). The former possibility is unlikely because of the structure of the cap, whereas it is well known that small changes in adsorbate coverage result in large variations of the work function (Kyritsakis \& Xanthakis, 2015; Lee et al., 1995). This effect is probably enhanced by the non-metallic character of the tip. Finally, since each tube has a unique tip, and hence a unique density of states at the tip, some tubes should be far more efficient emitters than others, depending on the position of the localized states with respect to the Fermi level. Some points are however not yet clarified, especially the behavior beyond the saturation point and the abrupt increase in the $I-V$ characteristics some single MWNTs. The nature of the current saturation phenomenon with stable charge distribution on the emitter surface is not fully understood. Amongst other possible explanations, it could indicate a new field emission mechanism. Further research will be needed to clarify this point. Nonetheless, it appears that such tips might provide an electron source with stable supply mechanism of saturated current for technological application. All of the tips were able to serve rather high emission currents of up to 20 $\mu \mathrm{A}$ or more without being destroyed. This indicates protective properties of the glass microemitters with internal MWCNTS.

\section{CONFLICT OF INTEREST}

No potential conflict of interest relevant to this article was reported.

\section{ACKNOWLEDGMENTS}

Emad Bani Ali would like to thank the Scientific Research Support Fund (SRSF) for their support. The authors would like to thank Deanship of the Academic Research at Mu'tah University for supporting this work. Authors acknowledge Prof. Dr. Saadi Abdul Jawad for providing the Nanocly ${ }^{\mathrm{TM}} \mathrm{NC}$ 7000 (MWCNTs).

\section{REFERENCES}

Al-Qudah M A, Mousa M S, and Fischer A (2015) Effect of insulating layer on the field electron emission performance of nano-apex metallic emitters. Mater. Sci. Eng. 92, 012021.

Bonard J M, Croci M, Klinke C, Kurt R, Noury O, and Weiss N (2002) Carbon nanotube films as electron field emitters. Carbon 40, 17151728.

Bonard J M, Maier F, Stockli T, Chatelain A, de Heer W A, Salvetat J P, and Forro $L$ (1998) Field emission properties of multiwalled carbon nanotubes. Ultramicroscopy 73, 7-15.

Bonard J M, Salvetat J P, Stockli T, Forro L, and Chatelain A (1999) Field emission from carbon nanotubes: perspectives for applications and clues to the emission mechanism. Appl. Phys. A 69, 245-254.

Choi W B, Chung D S, Lee J H, Jung J E, Lee N S, Park G S, and Kim J M (1999) Fully sealed, high-brightness carbon-nanotube field-emission display. Appl. Phys. Lett. 75, 3129-3131.

de Jonge $\mathrm{N}$ and Bonard J M (2004) Carbon nanotube electron sources and applications. Phil. Trans. R. Soc. Lond. A 362, 2239-2266.

Forbes R G (2012) Extraction of emission parameters for large-area field emitters, using a technically comlete Fowler-Nordheim-type equation. Nanotechnology 23, 095706.

Forbes R G, Deane J H B, Fischer A, and Mousa M S (2015) FowlerNordheim plot analysis: a progress report. Jordan J. Phys. 8, 125147.

Gröning O, Küttel O M, Emmenegger C, Gröning P, and Schlapbach L (2000) Field emission properties of carbon nanotubes. J. Vac. Sci. Technol. B 18, 665-678.

lijima S (1991) Helical microtubles of graphitic carbon. Nature 354, 5658.

Kim W K (2013) Ambient variable pressure field emission scanning electron microscopy for trichome profiling of plectranthus tomentosaby secondary electron imaging. Appl. Microsc. 43, 34-39.
Kyritsakis A and Xanthakis J P (2015) Derivation of a generalized FowlerNordheim equation for nanoscopic field-emitters. Proc. R. Soc. A 471, 20140811.

Latham R V and Mousa M S (1986) Hot electron emission from composite metal-insulator micropoint cathodes. Appl. Phys. 19, 699713.

Latham R V and Salim M A (1987) A microfocus cathode ray tube using an externally stabilized carbon-fibre field-emitting source. J. Phys. E.: Sci. Instrum. 20, 181-188.

Lee S C, Irokawa Y, Inoue M, and Shimizu R (1995) Behavior of zirconium in the $\mathrm{Zr}-\mathrm{O} / \mathrm{W}(\mathrm{IOO})$ system at high temperature, studied by ISS, AES and work-function measurements. Surf. Sci. 330, 289-296.

Madanat M A, Mousa M S, Al-Rabadi A N, and Fisher A (2015) Electron microscopy-based performance evaluation of various tungsten fieldemitter tips apex radii. Jordan J. Phys. 8, 79-85.

Moran Meza J A, Lubin C, Thoyer F, Villegas Rosales L A, Guitarra Espinoza A A, Martin F, and County J (2015) Fabrication of ultra-sharp tips from carbon fiber for scanning tunneling microscopy investigations of epitaxial graphene on 6H-SiC(0001) surface. Carbon 86, 363-370.

Mousa M S (1991) Effect of an internally conductive coating on the electron emission from glass tips. Surf. Sci. 246, 79-86.

Mousa M S, Alnawasreh S, Madanat M A, and Al-Rabadi A N (2015) Investigation of the field emission performance on nano-apexcarbon fiber and tungesten tips. Mater. Sci. Eng. 92, 12-22.

Mousa M S and Hibbert D B (1993a) Field emission of electrons from glass tips with internal conducting coats. J. Phys. D: Appl. Phys. 26, 697-703.

Mousa M S and Hibbert D B (1993b) Analysis of some properties of metal-glass microemitters subjected to strong electric field. Appl. Surf. Sci. 67, 59-65.

Saito R, Dresselhaus G, and Dresselhaus M S (1998) Physical Properties of Carbon Nanotubes (Imperial College Press, London). 
Saito $Y$ and Uemura S (2000) Field emission from carbon nanotubes and its application to electron sources. Carbon 38, 169-182.

Swanson L W and Bell A E (1973) Recent advances in field electron microscopy of metals. Adv. Electron. Electron Phys. 23, 193-309.
Thien V B, Garcia N, and Purcell S T (1996) Electron field emission from atom-sources: fabrication, properties, and applications of nanotips. Adv. Imaging Electron Phys. 95, 63-82. 